\title{
INITIAL COEFFICIENTS AND FOURTH HANKEL DETERMINANT FOR CERTAIN ANALYTIC FUNCTIONS
}

\author{
NAK EUN CHO AND VIRENDRA KUMAR \\ Dedicated to Professor V. Ravichandran \\ Received 21 October, 2019
}

\begin{abstract}
The present work is an attempt to give partial proofs of certain conjectures on the fifth coefficient of certain normalized analytic functions. Further, bounds on the sixth and seventh coefficients for the starlike functions related to a lune are also investigated. The non-sharp bound on third and fourth Hankel determinants are also obtained.
\end{abstract}

2010 Mathematics Subject Classification: 30C45; 30C50; 30C80

Keywords: starlike functions, third Hankel determinant, fourth Hankel determinant, initial coefficients

\section{INTRODUCTION}

Let $\mathcal{A}$ be the class of analytic functions $f$ defined on the unit disk $\mathbb{D}:=\{z: z \in$ $\mathbb{C}$ and $|z|<1\}$ and satisfying the conditions $f(0)=0=f^{\prime}(0)-1$. Thus the functions in the class $\mathcal{A}$ has the form $f(z)=z+\sum_{n=2}^{\infty} a_{n} z^{n}$. The collection of functions in $\mathcal{A}$, which are one-one, is denoted by $\mathcal{S}$. It was proved, in 1985, by de Branges that "if $f \in$ $\mathcal{S}$, then $\left|a_{n}\right| \leq n$." The bound $\left|a_{n}\right| \leq 2(1-\alpha), 0 \leq \alpha<1$, holds for the class of starlike and functions $\mathcal{S}^{*}(\alpha):=\left\{f: f \in \mathcal{S}\right.$ and $\left.\Re\left(z f^{\prime}(z) / f(z)\right)>\alpha\right\}$ of order $\alpha$. However, for the class of convex functions $\mathcal{K}(\alpha):=\left\{f: f \in \mathcal{S}\right.$ and $\left.1+\Re\left(z f^{\prime \prime}(z) / f^{\prime}(z)\right)>\alpha\right\}$ of order $\alpha$, we have the bound $\left|a_{n}\right| \leq 2(1-\alpha), 0 \leq \alpha<1$. The classes $\mathcal{S}^{*}:=$ $\mathcal{S}^{*}(0)$ and $\mathcal{K}:=\mathcal{K}(0)$ are known, respectively, as the classes of starlike and convex functions in $\mathbb{D}$. Finding bound on the coefficients of functions in a prescribed class has been one among the major problem in geometric function theory as they affects geometric properties. For example, the bound on the second coefficient gives the growth and distortion properties.

The Hankel determinant of the sequence of coefficients $a_{i}$ of functions in a given class also gives the important information about the function. For given positive integers $n$ and $q$, the Hankel determinant $H_{q, n}(f)$ related to the function $f \in \mathcal{A}$ is

Corresponding author: Virendra Kumar. 
given by

$$
H_{q, n}(f):=\left|\begin{array}{cccc}
a_{n} & a_{n+1} & \cdots & a_{n+q-1} \\
a_{n+1} & a_{n+2} & \cdots & a_{n+q} \\
\vdots & \vdots & \vdots & \vdots \\
a_{n+q-1} & a_{n+q} & \cdots & a_{n+2(q-1)}
\end{array}\right|, \quad a_{1}=1 .
$$

For some special choices of $n$ and $q ; H_{2,1}(f)=a_{3}-a_{2}^{2}$ is the famed Fekete-Szegó functional; $H_{2,2}(f)=a_{2} a_{4}-a_{3}^{2}$ is the second Hankel determinant, and $H_{3,1}(f)=$ $a_{3}\left(a_{2} a_{4}-a_{3}^{2}\right)-a_{4}\left(a_{4}-a_{2} a_{3}\right)+a_{5}\left(a_{3}-a_{2}^{2}\right)$ is the third Hankel determinant. The Hankel determinant $H_{q, n}(f)$ for the class $\mathcal{S}$ was investigated by Pommerenke [21] and Hayman [6]. There has been substantial progress in finding the bounds on the Hankel determinant for subclasses of $\mathcal{S}$, see[13].

In 2007, Janteng et al. [7] found the sharp estimate on the second Hankel determinant for the classes of starlike and convex functions. Non-sharp estimate on third Hankel determinant for classes of starlike and convex functions were investigated by Zaprawa [33] as $\left|H_{3,1}(f)\right| \leq 1$ and $\left|H_{3,1}(f)\right| \leq 49 / 540$, respectively. Kowalczyk et al. [8], in 2018, proved that the bound $\left|H_{3,1}(f)\right| \leq 4 / 135$ is sharp for the class of convex functions. However, the best known estimate for starlike functions is $\left|H_{3,1}(f)\right| \leq$ 4/135 due to Kwon et al. [11]. Later, in 2018, Lecko et al. [12] found the sharp bound $\left|H_{3,1}(f)\right| \leq 1 / 9$ for starlike function of order 1/2. Kowalczyk et al. [9] also found sharp bound of the third kind for the class $T(\alpha):=\{f \in \mathcal{A}: \Re(f(z) / z)>\alpha ; z \in \mathbb{D}\}$ for the cases when $\alpha=0$ and $\alpha=1 / 2$.

In 2015, Raina and Sokół [24] introduced the class

$$
\mathcal{S}_{q}^{*}:=\left\{f \in \mathcal{S}^{*}: \frac{z f^{\prime}(z)}{f(z)} \prec \sqrt{1+z^{2}}+z=: q(z)\right\}
$$

and discussed several properties of the class $\mathcal{S}_{q}^{*}$. Later, Raina and Sokół [23] conjectured that $\left|a_{4}\right| \leq 5 / 12,\left|a_{5}\right| \leq 2 / 9$ and $\left|a_{2} a_{4}-a_{3}^{2}\right| \leq 7 / 48$. In 2017, Cho et al. [Coefficient bounds for certain subclasses of starlike functions, preprint] settled the conjecture $\left|a_{4}\right| \leq 5 / 12$ posed by Raina and Sokòł [23] for functions in the class $\mathcal{S}_{q}^{*}$.

Recently, Çağlar et al. [3] obtained upper bound for the second Hankel determinant for certain subclasses of analytic and bi-univalent functions. Later, Srivastava et al. [30] also discussed several properties of a newly-constructed subclass of biunivalent functions in $\mathbb{D}$ defined by using a symmetric basic (or $q$-) derivative operator. Further, they investigated bound on $\left|a_{2} a_{4}-a_{3}^{2}\right|$. In [29] authors discussed bound on the Hankel determinant and the Toeplitz matrices for certain classes of analytic $q$ starlike functions. The authors [28] obtained non-sharp bound of the third Hankel determinant for a subclass of close-to-convex functions associated with the lemniscate of Bernoulli. They also investigated sharp bound on the initial coefficients. For more related results one may refer to the work $[5,16,26,27,31]$. 
Obradović and Ponnusamy [17] introduced the class

$$
\mathcal{U}(\lambda):=\left\{f \in \mathcal{A}:\left|\left(\frac{z}{f(z)}\right)^{2} f^{\prime}(z)-1\right|<\lambda, \lambda \in(0,1], z \in \mathbb{D}\right\} .
$$

The functions in the class $\mathcal{U}:=\mathcal{U}(0)$ are of the form $f(z)=z /(1-b z),|b| \leq 1$. Note that if $f \in \mathcal{U}(\lambda)$, then

$$
\frac{f(z)}{z} \prec \frac{1}{(1+z)(1+\lambda z)}, z \in \mathbb{D} .
$$

Obradović and co-authors have investigated several interesting properties of the class $\mathcal{U}(\lambda)$, see the works [17-19,22] and the references cited therein.

In 2016, Obradović et al. [18] conjectured that the $n^{\text {th }}$ coefficient of function $f \in$ $\mathcal{U}(\lambda)$ satisfies

$$
\left|a_{n}\right| \leq \sum_{k=0}^{n-1} \lambda^{k}(0<\lambda \leq 1, n \geq 2) .
$$

This conjecture has been verified for $n=2$ first in [32] and a simpler proof was given in [18]. More recently, Obradović et al. [19] proved the conjecture for $n=3,4$ with an alternate proof for the case $\mathrm{n}=2$, but it remains open for all $n \geq 5$. A partial solution to this conjecture was given in [22, Theorem 1, p. 91].

$$
\left|a_{n}\right| \leq 1+\lambda \sqrt{n-1} \sqrt{\sum_{k=0}^{n-2} \lambda^{2 k}} \quad(0<\lambda \leq 1, n \geq 2) .
$$

In the present paper, we improve the existing bound on $\left|a_{5}\right|$ given in the above inequality for certain range of $\lambda$ for $f \in \mathcal{U}(\lambda)$. We give non-sharp bound on $\left|a_{i}\right|$ $(i=5,6,7),\left|H_{3,1}(f)\right|$ and $\left|H_{4,1}(f)\right|$ for $f \in \mathcal{S}_{q}^{*}$.

\section{INITIAL COEFFICIENTS}

The following theorem gives a refinement and improvement to the bound on $\left|a_{5}\right|$ given in [22, Theorem 1, p. 91]:

Theorem 1. Let $f(z)=z+a_{2} z^{2}+a_{3} z^{3}+\cdots \in \mathcal{U}(\lambda)$. Then

$$
\left|a_{5}\right| \leq \frac{1}{2}\left(2+2 \lambda+5 \lambda^{2}+6 \lambda^{3}+4 \lambda^{4}\right) .
$$

We need the following lemma to prove our result. Before proceeding further we recall that the class of functions $p(z)=1+\sum_{n=1}^{\infty} p_{n} z^{n}$ with $\Re p(z)>0, z \in \mathbb{D}$ is denoted by $\mathcal{P}$ and they are related with the Schwarz function $w: \mathbb{D} \rightarrow \mathbb{C}$ by means of the relation

$$
w(z)=\frac{p(z)-1}{p(z)+1} .
$$


Lemma 1 ([25], Lemma 2.3, p. 507). Let $p \in \mathcal{P}$. Then for all $n, m \in \mathbb{N}$,

$$
\left|\mu p_{n} p_{m}-p_{m+n}\right| \leq \begin{cases}2, & 0 \leq \mu \leq 1 \\ 2|2 \mu-1|, & \text { elsewhere. }\end{cases}
$$

If $0<\mu<1$, then the inequality is sharp for the function

$$
p(z)=\left(1+z^{m+n}\right) /\left(1-z^{m+n}\right) .
$$

In the other cases, the inequality is sharp for the function $\hat{p}_{0}$.

Proof of Theorem 1. Let $f \in \mathcal{U}(\lambda)$. Then, from [19, Eqn.(3)], we have

$$
\frac{f(z)}{z} \prec \frac{1}{(1+z)(1+\lambda z)} \text {. }
$$

Therefore, for $p(z)=1+\sum_{n=1}^{\infty} p_{n} z^{n}$ with $\Re p(z)>0, z \in \mathbb{D}$ such that

$$
\frac{f(z)}{z}=\frac{1}{\left(1+\frac{1-p(z)}{1+p(z)}\right)\left(1+\lambda \frac{1-p(z)}{1+p(z)}\right)} .
$$

On comparing coefficients, we have

$$
\begin{gathered}
a_{2}=-\frac{p_{1}}{2}(\lambda+1), \\
a_{3}=\frac{1}{4}\left(\left(\lambda^{2}+2 \lambda+2\right) p_{1}^{2}-2(\lambda+1) p_{2}\right), \\
a_{4}=\frac{1}{8}\left(-\left(\lambda^{3}+3 \lambda^{2}+4 \lambda+4\right) p_{1}^{3}+4\left(\lambda^{2}+2 \lambda+2\right) p_{1} p_{2}-4(\lambda+1) p_{3}\right)
\end{gathered}
$$

and

$$
\begin{aligned}
16 a_{5}= & -8(1+\lambda) p_{4}+4\left(2+2 \lambda+\lambda^{2}\right)+8\left(2+2 \lambda+\lambda^{2}\right) p_{1} p_{3} \\
& -6\left(4+4 \lambda+3 \lambda^{2}+\lambda^{3}\right) p_{1}^{2} p_{2}+\left(8+8 \lambda+7 \lambda^{2}+4 \lambda^{3}+\lambda^{4}\right) p_{1}^{4},
\end{aligned}
$$

where $A:=8+8 \lambda+7 \lambda^{2}+4 \lambda^{3}+\lambda^{4}$. Now rearranging the terms of (2.2), we have

$$
\begin{aligned}
\frac{16}{A} a_{5}= & -\frac{8(1+\lambda)}{A} p_{4}+\frac{4\left(2+2 \lambda+\lambda^{2}\right)}{A} p_{2}^{2}+\frac{8\left(2+2 \lambda+\lambda^{2}\right)}{A} p_{1} p_{3} \\
& -\frac{6\left(4+4 \lambda+3 \lambda^{2}+\lambda^{3}\right)}{A} p_{1}^{2} p_{2}+p_{1}^{4} .
\end{aligned}
$$

Now upon using triangle inequality, (2.3) gives

$$
\begin{aligned}
\left|\frac{16}{A} a_{5}\right| \leq & \left|p_{1}^{4}+2 p_{1} p_{3}+p_{2}^{2}-3 p_{1}^{2} p_{2}-p_{4}\right|+\left|\frac{3 \lambda^{2}(1+\lambda)^{2}}{A} p_{1}^{2} p_{2}-\frac{\left(3+4 \lambda+\lambda^{2}\right) \lambda^{2}}{A} p_{2}^{2}\right| \\
& +\left|\frac{\lambda^{2}\left(\lambda^{2}+4 \lambda+7\right)}{A} p_{4}-\frac{2 \lambda^{2}\left(3+4 \lambda+\lambda^{2}\right)}{A} p_{1} p_{3}\right|
\end{aligned}
$$


To estimate bound on $\left|a_{5}\right|$, now we consider all three terms in the right hand side of (2.4) one by one and find the bound on them. It is well known form [4] (see also [15, Lemma 3, p. 227]) that

$$
\left|p_{1}^{4}+2 p_{1} p_{3}+p_{2}^{2}-3 p_{1}^{2} p_{2}-p_{4}\right| \leq 2 .
$$

The following are obtained by using Lemma 1 :

$$
\begin{aligned}
\left|\frac{3 \lambda^{2}(1+\lambda)^{2}}{A} p_{1}^{2} p_{2}-\frac{\lambda^{2}\left(3+4 \lambda+\lambda^{2}\right)}{A} p_{2}^{2}\right| & =\left|\frac{\left(3+4 \lambda+\lambda^{2}\right) \lambda p_{2}}{A}\left(\frac{3(1+\lambda)^{2}}{3+4 \lambda+\lambda^{2}} p_{1}^{2}-p_{2}\right)\right| \\
& \leq \frac{4 \lambda^{2}\left(5 \lambda^{2}+8 \lambda+3\right)}{A} \\
\left|\frac{\lambda^{2}\left(\lambda^{2}+4 \lambda+7\right)}{A} p_{4}-\frac{2 \lambda^{2}\left(\lambda^{2}+4 \lambda+3\right)}{A}\right| & =\frac{\lambda^{2}}{A}\left|\left(\lambda^{2}+4 \lambda+3\right) p_{1} p_{3}-\left(\lambda^{2}+4 \lambda+7\right) p_{4}\right| \\
& =\frac{\left(\lambda^{2}+4 \lambda+7\right) \lambda^{2}}{A}\left|\frac{\left(\lambda^{2}+4 \lambda+3\right)}{\left(\lambda^{2}+4 \lambda+7\right)} p_{1} p_{3}-p_{4}\right| \\
& \leq \frac{2\left(\lambda^{2}+4 \lambda+7\right) \lambda^{2}}{A} .
\end{aligned}
$$

Now, from (2.4), (2.5), (2.6) and (2.7), we have

$$
\begin{aligned}
\left|a_{5}\right| & \leq 2 \frac{A}{16}+\left(\frac{4 \lambda^{2}\left(5 \lambda^{2}+8 \lambda+3\right)}{A}+\frac{2\left(\lambda^{2}+4 \lambda+7\right) \lambda^{2}}{A}\right) \\
& =\frac{A}{8}+\frac{4 \lambda^{2}\left(5 \lambda^{2}+8 \lambda+3\right)}{4}+\frac{2\left(\lambda^{2}+4 \lambda+7\right) \lambda^{2}}{8} \\
& =\frac{2+2 \lambda+5 \lambda^{2}+6 \lambda^{3}+4 \lambda^{4}}{2} .
\end{aligned}
$$

This completes the proof.

Remark 1. In [22, Theorem 1, p. 91], the following non-sharp bound was proved:

$$
\left|a_{5}\right| \leq 1+2 \lambda \sqrt{\left(\lambda^{2}+1\right)\left(\lambda^{4}+1\right)} .
$$

Now if $x_{0} \approx 0.305398$ is the root of $48 x^{5}+60 x^{4}+76 x^{3}+33 x^{2}+20 x-12=0$, then from (2.1) and (2.8), we have the following refined result:

$$
\left|a_{5}\right| \leq \begin{cases}\frac{2+2 \lambda+5 \lambda^{2}+6 \lambda^{3}+4 \lambda^{4}}{2}, & 0<\lambda \leq x_{0} \\ 1+2 \lambda \sqrt{\left(\lambda^{2}+1\right)\left(\lambda^{4}+1\right)}, & x_{0} \leq x<1 .\end{cases}
$$

Thus, we see that our result improves over the result proved in [22, Theorem 1, p. 91] for the range $0<\lambda \leq x_{0}$.

In the following theorem non-sharp bounds on $\left|a_{5}\right|,\left|a_{6}\right|$ and $\left|a_{7}\right|$ for the class $\mathcal{S}_{q}^{*}$ are investigated.

Theorem 2. Let $f(z)=z+a_{2} z^{2}+a_{3} z^{3}+\cdots \in \mathcal{S}_{q}^{*}$. Then 
(i) $\left|a_{5}\right| \leq \frac{1}{8}(1+2 \sqrt{2}) \approx 0.478553$,

(ii) $\left|a_{6}\right| \leq \frac{5(711+32 \sqrt{79})}{8532} \approx 0.341303$,

(iii) $\left|a_{7}\right| \leq \frac{2665}{2304} \approx 1.15668$.

We need the following lemmas to prove the above theorem:

Lemma 2 ([14], Theorem 4(b), p. 678). A function $p \in \mathcal{P}$ if and only if

$$
\sum_{j=0}^{\infty}\left\{\left|2 z_{j}+\sum_{k=1}^{\infty} p_{k} z_{k+j}\right|^{2}-\left|\sum_{k=0}^{\infty} p_{k+1} z_{k+j}\right|^{2}\right\} \geq 0
$$

for every sequence $\left\{z_{k}\right\}_{k=1}^{\infty}$ of complex numbers that satisfies $\lim _{k \rightarrow \infty}\left|z_{k}\right|^{1 / k}<1$.

Lemma 3 ([20], Proposition 6, p. 7). Let $\overline{\mathbb{D}}:=\{z \in \mathbb{C}:|z| \leqq 1\}$. Also, for any real numbers $a, b$ and $c$, let the quantity $Y(a, b, c):=\max _{z \in \overline{\mathbb{D}}}\left\{\left|a+b z+c z^{2}\right|+1-|z|^{2}\right\}$. If $a c \geqq 0$, then

$$
Y(a, b, c)= \begin{cases}|a|+|b|+|c| & (|b| \geqq 2(1-|c|)), \\ 1+|a|+\frac{b^{2}}{4(1-|c|)} & (|b|<2(1-|c|)) .\end{cases}
$$

Furthermore, if ac $<0$, then

$$
Y(a, b, c)= \begin{cases}1-|a|+\frac{b^{2}}{4(1-|c|)} & \left(-4 a c\left(c^{-2}-1\right) \leqq b^{2} ;|b|<2(1-|c|)\right) \\ 1+|a|+\frac{b^{2}}{4(1+|c|)} & \left(b^{2}<\min \left\{4(1+|c|)^{2},-4 a c\left(c^{-2}-1\right)\right\}\right) \\ R(a, b, c) & \text { (otherwise), }\end{cases}
$$

where

$$
R(a, b, c)= \begin{cases}|a|+|b|-|c| & (|c|(|b|+4|a|) \leqq|a b|) \\ -|a|+|b|+|c| & (|a b| \leqq|c|(|b|-4|a|)) \\ (|c|+|a|) \sqrt{1-\frac{b^{2}}{4 a c}} & \text { (otherwise) }\end{cases}
$$


Lemma 4 ([10], Lemma 1). Let $p(z)=1+p_{1} z+p_{2} z^{2}+p_{3} z^{3}+\cdots \in \mathcal{P}$. Then, for any real number $\mu$,

$$
\left|\mu p_{3}-p_{1}^{3}\right| \leq \begin{cases}2|\mu-4| & \left(\mu \leq \frac{4}{3}\right) \\ 2 \mu \sqrt{\frac{\mu}{\mu-1}} & \left(\frac{4}{3}<\mu\right) .\end{cases}
$$

The result is sharp.

Lemma 5 ([1], Corollary 1, p. 68). Let $p \in \mathcal{P}$. Then

$$
\left|p_{3}-(\mu+1) p_{1} p_{2}+\mu p_{1}^{3}\right| \leq \begin{cases}2, & 0 \leq \mu \leq 1 ; \\ 2|\mu-1|, & \text { elsewhere. }\end{cases}
$$

Proof of Theorem 2. (i) Since $f \in \mathcal{S}_{q}^{*}$, it follows that there exists a Schwarz function $w$, such that

$$
\frac{z f^{\prime}(z)}{f(z)}=w(z)+\sqrt{1+w^{2}(z)} .
$$

Writing

$$
w(z)=\frac{p(z)-1}{p(z)+1}
$$

with $p(z)=1+p_{1} z+p_{2} z^{2}+p_{3} z^{3}+\cdots \in \mathcal{P}$, and equating the coefficients of like power terms, we have

$$
a_{5}=\frac{1}{384}\left(p_{1}^{4}-10 p_{1}^{2} p_{2}+8 p_{1} p_{3}+48 p_{4}\right)
$$

and therefore,

$$
\begin{aligned}
\left|a_{5}\right| & =\frac{1}{384}\left|p_{1}^{4}-10 p_{1}^{2} p_{2}+8 p_{1} p_{3}+48 p_{4}\right| \\
& \leq \frac{1}{384}\left(\left|48 p_{4}-10 p_{1}^{2} p_{2}\right|+\left|p_{1}^{4}+8 p_{1} p_{3}\right|\right) \\
& \leq \frac{1}{384}\left(48+\left|48 p_{4}-10 p_{1}^{2} p_{2}\right|\right) .
\end{aligned}
$$

We now choose a sequence

$$
z_{0}=\frac{5}{24} p_{1} p_{2}, z_{1}=0, z_{2}=0, z_{3}=-1, z_{k}=0(k \geq 4)
$$

to estimate the maximum value of $\left|48 p_{4}-10 p_{1}^{2} p_{2}\right|$.

Now an application of Lemma 2, for the above choice of sequence gives

$$
\left|\frac{5}{24} p_{1}^{2} p_{2}-p_{4}\right|^{2} \leq\left|p_{3}-\frac{5}{12} p_{1} p_{2}\right|^{2}+4 \leq 8 .
$$


Therefore,

$$
\left|10 p_{1}^{2} p_{2}-48 p_{4}\right| \leq 96 \sqrt{2}
$$

and from Eq. (2.11), we have

$$
\left|a_{5}\right| \leq \frac{1}{8}(1+2 \sqrt{2}) \approx 0.478553
$$

This was the desired bound on $\left|a_{5}\right|$.

(ii) Proceeding as above, we have

$$
a_{6}=\frac{-p_{1}^{5}+42 p_{1}^{3} p_{2}-80 p_{1}^{2} p_{3}-80 p_{1} p_{2}^{2}+48 p_{1} p_{4}-32 p_{2} p_{3}+384 p_{5}}{3840}
$$

and

where

$$
a_{7}=\frac{T}{184320}
$$

$$
\begin{aligned}
T:= & -59 p_{1}^{6}-392 p_{1}^{4} p_{2}+1840 p_{1}^{3} p_{3}+32 p_{1}^{2}\left(95 p_{2}^{2}-99 p_{4}\right) \\
& -64 p_{1}\left(97 p_{2} p_{3}+96 p_{5}\right)-320\left(3 p_{2}^{3}+6 p_{2} p_{4}+4 p_{3}^{2}-48 p_{6}\right) .
\end{aligned}
$$

To find the estimate on $\left|a_{6}\right|$, by suitably rearranging the terms of (2.12) and using the triangle inequality, we have:

$$
\begin{aligned}
& 3840\left|a_{6}\right|=\left|-p_{1}^{5}+42 p_{1}^{3} p_{2}-80 p_{1}^{2} p_{3}-80 p_{1} p_{2}^{2}+48 p_{1} p_{4}-32 p_{2} p_{3}+384 p_{5}\right| \\
& =\left|-80 p_{1}^{2}\left(p_{3}-\frac{21}{40} p_{1} p_{2}+\frac{19}{40} p_{1}^{3}\right)+\left(48 p_{1} p_{4}-80 p_{1} p_{2}^{2}\right)+\left(384 p_{5}-32 p_{2} p_{3}\right)+37 p_{1}^{5}\right| \\
& \leq 80\left|p_{1}^{2}\left(p_{3}-\frac{21}{40} p_{1} p_{2}+\frac{19}{40} p_{1}^{3}\right)\right|+\left|48 p_{1} p_{4}-80 p_{1} p_{2}^{2}\right|+\left|384 p_{5}-32 p_{2} p_{3}\right|+37\left|p_{1}\right|^{5} .
\end{aligned}
$$

Now using Lemma 1, Lemma 5 and the fact $\left|p_{i}\right| \leq 2$, we have

$$
80\left|p_{1}^{2}\left(p_{3}-\frac{21}{40} p_{1} p_{2}+\frac{19}{40} p_{1}^{3}\right)\right| \leq 640,\left|48 p_{1} p_{4}-80 p_{1} p_{2}^{2}\right| \leq 320
$$

and

$$
\left|384 p_{5}-32 p_{2} p_{3}\right|=384\left|\frac{1}{12} p_{2} p_{3}-p_{5}\right| \leq 768 .
$$

Now from (2.15), (2.16) and (2.17), we have

$$
\left|a_{6}\right| \leq \frac{2272}{8532} \approx 0.341303 \text {. }
$$

This was the desired estimate on $\left|a_{6}\right|$.

(iii) From (2.14), by suitably rearranging the terms, we have

$$
\begin{aligned}
184320 a_{7}= & -59 p_{1}^{6}-392 p_{1}^{4} p_{2}+1840 p_{1}^{3} p_{3}+32 p_{1}^{2}\left(95 p_{2}^{2}-99 p_{4}\right) \\
& -64 p_{1}\left(97 p_{2} p_{3}+96 p_{5}\right)-320\left(3 p_{2}^{3}+6 p_{2} p_{4}+4 p_{3}^{2}-48 p_{6}\right)
\end{aligned}
$$


or

$$
\begin{aligned}
184320\left|a_{7}\right| \leq & 320\left|6 p_{2} p_{4}-48 p_{6}\right|+\left|-392 p_{1}^{4} p_{2}+1840 p_{1}^{3} p_{3}\right| \\
& +\left|-3040 p_{1}^{2} p_{2}^{2}+960 p_{2}^{3}\right|+\left|59 p_{1}^{6}+3168 p_{1}^{2} p_{4}\right| \\
& +\left|64 p_{1}\left(97 p_{2} p_{3}+96 p_{5}\right)\right|+\left|2180 p_{3}^{2}\right| .
\end{aligned}
$$

Now applications of Lemma 1 and the fact $\left|p_{i}\right| \leq 2$, gives

$$
\begin{gathered}
320\left|6 p_{2} p_{4}-48 p_{6}\right| \leq 30720,\left|-392 p_{1}^{4} p_{2}+1840 p_{1}^{3} p_{3}\right| \leq 29440, \\
\left|-3040 p_{1}^{2} p_{2}^{2}+960 p_{2}^{3}\right| \leq 40960 .
\end{gathered}
$$

and

$$
\left|59 p_{1}^{6}+3168 p_{1}^{2} p_{4}\right|+\left|64 p_{1}\left(97 p_{2} p_{3}+96 p_{5}\right)\right|+\left|2180 p_{3}^{2}\right| \leq 108480 .
$$

Now from (2.18), (2.19), (2.20) and (2.21), we have

This completes the proof.

$$
\left|a_{7}\right| \leq \frac{2665}{2304} \approx 1.15668 \text {. }
$$

\section{Third And Fourth Hankel Determinants}

\subsection{Third Hankel determinant}

In this section, we find an estimate on the third Hankel determinant for functions in the class $\mathcal{S}_{q}^{*}$. As before, for functions in the class $\mathcal{S}_{q}^{*}$, we have

$$
a_{2}=\frac{p_{1}}{2}, \quad a_{3}=\frac{1}{16}\left(p_{1}^{2}+4 p_{2}\right) \quad \text { and } \quad a_{4}=\frac{1}{96}\left(16 p_{3}+4 p_{1} p_{2}-p_{1}^{3}\right) .
$$

A computation using (2.10), (2.12), (2.13) and (3.1), gives

$$
H_{3,1}(f)=a_{3}\left(a_{2} a_{4}-a_{3}^{2}\right)-a_{4}\left(a_{4}-a_{2} a_{3}\right)+a_{5}\left(a_{3}-a_{2}^{2}\right)=\frac{S}{36864},
$$

where

$$
\begin{aligned}
S:= & -55 p_{1}^{6}+128 p_{1}^{4} p_{2}+368 p_{1}^{3} p_{3}+1216 p_{1} p_{2} p_{3} \\
& -32 p_{1}^{2}\left(11 p_{2}^{2}+27 p_{4}\right)-64\left(9 p_{2}^{3}+16 p_{3}^{2}-18 p_{2} p_{4}\right) .
\end{aligned}
$$

By rearranging terms and using the triangle inequality from (3.2), we have

$$
\begin{aligned}
36864\left|H_{3,1}(f)\right| \leq & \left|-55 p_{1}^{6}+128 p_{1}^{4} p_{2}\right|+\left|1152 p_{2} p_{4}-864 p_{1}^{2} p_{4}\right| \\
& +\left|1216 p_{1} p_{2} p_{3}-1024 p_{3}^{2}\right|+\left|368 p_{1}^{3} p_{3}-352 p_{1}^{2} p_{2}^{2}\right|+\left|-576 p_{2}^{3}\right| .
\end{aligned}
$$

Using Lemma 1 and the fact $\left|p_{i}\right| \leq 2$, we have

$$
\begin{gathered}
\left|-55 p_{1}^{6}+128 p_{1}^{4} p_{2}\right| \leq 4096, \quad\left|1152 p_{2} p_{4}-864 p_{1}^{2} p_{4}\right| \leq 4608, \\
\left|1216 p_{1} p_{2} p_{3}-1024 p_{3}^{2}\right| \leq 5632 \text { and }\left|-576 p_{2}^{3}\right| \leq 4608 .
\end{gathered}
$$


Now in order to get the desired bound, we claim that $\left|368 p_{1}^{3} p_{3}-352 p_{1}^{2} p_{2}^{2}\right| \leq 5632$. To establish our claim, we shall prove that

$$
\left|\frac{23}{22} p_{1} p_{3}-p_{2}^{2}\right| \leq 4
$$

We use the following:

Lemma 6 ([15], Libera and Zlotkiewicz). Let $p(z)=1+p_{1} z+p_{2} z^{2}+\cdots \in \mathcal{P}$ with $p_{1} \geq 0$. Then

$$
2 p_{2}=p_{1}^{2}+x\left(4-p_{1}^{2}\right)
$$

and

$$
4 p_{3}=p_{1}^{3}+2 p_{1}\left(4-p_{1}^{2}\right) x-p_{1}\left(4-p_{1}^{2}\right) x^{2}+2\left(4-p_{1}^{2}\right)\left(1-|x|^{2}\right) y
$$

for some $x$ and $y$ such that $|x| \leq 1$ and $|y| \leq 1$.

To prove (3.6), we consider $(23 / 22) p_{1} p_{3}-p_{2}^{2}$. After substituting expression for $p_{2}, p_{3}$ and simplifying, from Lemma 6 , we have

$$
\begin{aligned}
I:= & \frac{23}{22} p_{1} p_{3}-p_{2}^{2} \\
= & \frac{23}{88} p_{1}\left(p_{1}^{3}+2 p_{1}\left(4-p_{1}^{2}\right) x-p_{1}\left(4-p_{1}^{2}\right) x^{2}+2\left(4-p_{1}^{2}\right)\left(1-|x|^{2}\right)\right. \\
& \left.-\frac{1}{4}\left(p_{1}+x\left(4-p_{1}^{2}\right)\right)^{2}\right) .
\end{aligned}
$$

Using the invariant property for the class $\mathcal{S}_{q}^{*}$ under rotation, we can assume that $s:=p_{1} \in[0,2]$ and thus (3.9) becomes

$$
I=\frac{1}{88} s^{4}+\frac{1}{88}\left(4-s^{2}\right) \frac{s^{2}}{2}+\frac{1}{4}\left(4-s^{2}\right)\left(\frac{45}{22} s^{2}-4\right) x^{2}+\frac{23}{44} s\left(4-s^{2}\right)\left(1-|x|^{2}\right) y .
$$

Now from (3.10), we have

$$
I=\left\{\begin{array}{cc}
4, & s=0 \\
\frac{6}{11}, & s=2 .
\end{array}\right.
$$

For $s \in(0,2)$ consider the expression:

$$
I=\frac{23 s}{44}\left(4-s^{2}\right)\left(a+b x+c x^{2}+1-|x|^{2}\right),
$$

where

$$
a:=\frac{s^{3}}{23\left(4-s^{2}\right)}, b:=\frac{s}{23} \text { and } c:=\frac{45 s^{2}-88}{46 s} .
$$

For suitability, we divide the calculation in five cases:

Case (I): Here $a>0$ always and $c \geq 0$ if and only if $1.39841 \approx(2 / 3) \sqrt{22 / 5}=$ : 
$s_{1} \leq s<2$. The condition $|b|>2(1-|c|)=2(1-|c|)$ holds if $s \geq 1.97073 \approx$ $(23+\sqrt{4577}) / 46=: s_{2}$. Thus, for $s \in\left[s_{2}, 2\right)$, we have

$$
I=\frac{23 s}{44}\left(4-s^{2}\right)(|a|+|b|+|c|)=\frac{\left(4-s^{2}\right)\left(45 s^{4}-276 s^{2}+352\right)}{88\left(s^{2}-4\right)}=: f_{1}(s) .
$$

Now computation shows that $f_{1}$ attains its maximum at $s_{0}=\sqrt{46 / 15}$ and

$$
f_{1}(s) \leq f_{1}\left(s_{0}\right)=\frac{89}{110}, \quad s \in\left(s_{1}, 2\right) .
$$

Case (II): The condition $|b|<2(1-|c|)=2(1-c)$ holds for $s \in\left[s_{1}, s_{2}\right)$ and for this range, we have

$$
I=\frac{s\left(91 s^{4}-1980 s^{3}-2024 s^{2}+8280 s+8096\right)}{88(45 s+44)}=f_{2}(s) .
$$

Computation reveals that $f_{2}$ never vanish in $\left[s_{1}, s_{2}\right)$, therefore,

$$
f_{2}(s) \leq \max \left\{f_{2}\left(s_{1}\right), f_{2}\left(s_{2}\right)\right\}=f_{1}\left(s_{1}\right)=\frac{2(14520+23827 \sqrt{110})}{334125} \approx 1.58276 .
$$

Case (III): Now computation shows that

$$
\min \left\{4\left(1+c^{2}\right),-4 a c\left(c^{-2}-1\right)\right\}= \begin{cases}-4 a c\left(c^{-2}-1\right), & 0<s \leq s_{3} ; \\ 4\left(1+c^{2}\right), & s_{3}<s<s_{1},\end{cases}
$$

where $s_{3}$ is a root of $95175 x^{6}-443252 x^{4}+859232 x^{2}-681472=0$ in $\left(0, s_{1}\right)$. Further, $b^{2}<-4 a c\left(c^{-2}-1\right)$ holds if and only if

$$
\frac{s^{2}}{529} \leq-\frac{2 s^{2}\left(2025 s^{2}-1936\right)}{529\left(45 s^{2}-88\right)}
$$

or equivalently

$$
0.983378 \approx 2 \sqrt{\frac{22}{91}}=: s_{4}=<s<s_{1} .
$$

Moreover, $b^{2}<4\left(1+c^{2}\right)$ holds for all $s>0$. Thus, for $s \in\left(s_{4}, s_{1}\right)$, we have

$$
\begin{gathered}
I=\frac{s\left(91 s^{4}-1980 s^{3}-2024 s^{2}+8280 s+8096\right)}{88(45 s+44)}=f_{3}(s) . \\
f_{3}^{\prime}(s)=0 \text { for } s=s^{*} \approx 1.1977 \in\left(s_{4}, s_{1}\right) \text { and } f_{3}^{\prime \prime}\left(s^{*}\right)<0, \text { so } \\
f_{3}(s) \leq \max \left\{f_{3}\left(s^{*}\right), f_{3}\left(s_{1}\right), f_{3}(4)\right\}=f_{3}\left(s^{*}\right) \approx 1.65372, \quad s \in\left(s_{4}, s_{1}\right) .
\end{gathered}
$$

Case (IV): It can be verified that $a b \leq-c(b-4 a)$ holds for $s \in\left(0, s_{5}\right]$, where

$$
s_{5}:=\sqrt{\frac{2}{223}(155-3 \sqrt{489})} .
$$


In this case, we have

$$
I=\frac{41 s^{4}}{88}-\frac{65 s^{2}}{22}+4=: f_{4}(s) .
$$

Now a computation shows that, for $s \in\left(0, s_{5}\right]$,

$$
\begin{aligned}
f_{4}(s) & \leq \max \left\{f_{4}(0), f_{4}\left(s_{5}\right)\right\} \\
& =f_{4}\left(s_{5}\right) \\
& =\frac{4(11911+555 \sqrt{489})}{49729} \approx 1.94526 .
\end{aligned}
$$

Case (V): In view of Lemma 3, for the case, $s \in\left(s_{5}, s_{4}\right]$, we have

$$
I=\frac{1}{88} \sqrt{\frac{180-91 s^{2}}{176-90 s^{2}}}\left(47 s^{4}-268 s^{2}+352\right)=: f_{5}(s) .
$$

Now a computation shows that

$$
\begin{aligned}
f_{5}(s) & \leq \max \left\{f_{5}\left(s_{4}\right), f_{5}\left(s_{5}\right)\right\}=f_{5}\left(s_{5}\right) \\
& =\frac{\sqrt{4930+138 \sqrt{489}}(6139+261 \sqrt{489})}{547019} \approx 1.94526, s \in\left(s_{5}, s_{4}\right] .
\end{aligned}
$$

From (3.11)-(3.16), we conclude that $I \leq 4$, that is

$$
\left|\frac{23}{22} p_{1} p_{3}-p_{2}^{2}\right| \leq 4
$$

The above inequality (3.17) is sharp for the function $p \tilde{(z)}=\left(1+z^{3}\right) /\left(1-z^{3}\right)$ and therefore,

$$
\left|368 p_{1}^{3} p_{3}-352 p_{1}^{2} p_{2}^{2}\right|=352\left|p_{1}^{2}\right|\left|\frac{23}{22} p_{1} p_{3}-p_{2}^{2}\right| \leq 5632 .
$$

From (3.3), (3.4), (3.5) and (3.18), we have

$$
\left|H_{3,1}(f)\right| \leq \frac{2}{3} \approx 0.67 \text {. }
$$

Thus, we have the following:

Theorem 3. Let $f(z)=z+a_{2} z^{2}+a_{3} z^{3}+\cdots \in S_{q}^{*}$. Then

$$
\left|H_{3,1}(f)\right| \leq \frac{2}{3} \approx 0.67 .
$$




\subsection{Fourth Hankel determinant}

The fourth Hankel determinant can be expressed as, see also [2]:

$$
H_{4,1}(f)=a_{7} H_{3,1}(f)-a_{6} \Delta_{1}+a_{5} \Delta_{2}-a_{4} \Delta_{3},
$$

where

$$
\begin{aligned}
& \Delta_{1}=a_{3} a_{6}-a_{4} a_{5}-a_{2}\left(a_{2} a_{6}-a_{3} a_{5}\right)+a_{4}\left(a_{2} a_{4}-a_{3}^{2}\right), \\
& \Delta_{2}=\left(a_{4} a_{6}-a_{5}^{2}\right)-a_{2}\left(a_{3} a_{6}-a_{4} a_{5}\right)+a_{3}\left(a_{3} a_{5}-a_{4}^{2}\right)
\end{aligned}
$$

and

$$
\Delta_{3}=a_{2}\left(a_{4} a_{6}-a_{5}^{2}\right)-a_{3}\left(a_{3} a_{6}-a_{4} a_{5}\right)+a_{4}\left(a_{3} a_{5}-a_{4}^{2}\right) .
$$

To compute the fourth Hankel determinant for the functions in class $\mathcal{S}_{q}^{*}$, we substitute the vales of $a_{i}$ in terms of $p_{j}$ as given in (2.10), (2.12), (2.13) and (3.1) and after simplification we have

$$
\begin{aligned}
122880 \Delta_{1}= & 31 p_{1}^{7}-400 p_{1}^{5} p_{2}+240 p_{1}^{4} p_{3}+32 p_{1}^{3}\left(18 p_{2}^{2}+11 p_{4}\right) \\
& -64 p_{1}\left(15 p_{2}^{3}-20 p_{3}^{2}-26 p_{2} p_{4}\right)+256 p_{1}^{2}\left(2 p_{2} p_{3}-9 p_{5}\right) \\
& -512\left(3 p_{2}^{2} p 3+5 p_{3} p_{4}-6 p_{2} p_{5}\right) .
\end{aligned}
$$

Rearranging the terms of (3.19) and applying Lemmas 1 and 4, we have

$$
\begin{aligned}
122880\left|\Delta_{1}\right| \leq & \left|31 p_{1}^{7}-400 p_{1}^{5} p_{2}\right|+\left|576 p_{1}^{3} p_{2}^{2}-960 p_{1} p_{2}^{3}\right|+\left|3072 p_{2} p_{5}-1536 p_{2}^{2} p_{3}\right| \\
& +256 \mid p_{1}^{2}\left(2 p_{2} p_{3}-9 p_{5}|+| 352 p_{1}^{3} p_{4}-2560 p_{3} p_{4} \mid\right. \\
& +64\left|p_{1}\right|\left|20 p_{3}^{2} p_{1}+26 p_{2} p_{4}\right| \\
\leq & \frac{1024}{69}(5451+40 \sqrt{345}) .
\end{aligned}
$$

Therefore, we have

$$
\left|\Delta_{1}\right| \leq \frac{5451+40 \sqrt{345}}{8280} \approx 0.748064
$$

In a similar line, we have

$$
\begin{aligned}
-491520 \Delta_{2}= & \left(-552 p_{1}^{5} p_{3}+3 p_{1}^{8}\right)+\left(656 p_{4} p_{1}^{4}-576 p_{1}^{2} p_{2} p_{4}\right) \\
& +\left(-1920 p_{1}^{3} p_{2} p_{3}+1216 p_{1} p_{2}^{2} p_{3}\right)+\left(7680 p_{4}^{2}-3584 p_{1} p_{3} p_{4}\right) \\
& +\left(4096 p_{1}^{2} p_{2} p_{5}-8192 p_{3} p_{5}\right)+\left(1216 p_{1} p_{2}^{2} p_{3}-3840 p_{2}^{2} p_{4}\right) .
\end{aligned}
$$

From (3.21) and applying Lemmas 1 and 4 , we have

$$
\left|\Delta_{2}\right| \leq \frac{118279+92 \sqrt{8418}}{117120} \approx 1.08197
$$

Similarly rearranging the terms of

$$
8847360 \Delta_{3}=-29 p_{1}^{9}-78 p_{1}^{7} p_{2}+768 p_{1}^{6} p_{3}-72 p_{1}^{5}\left(17 p_{2}^{2}+74 p_{4}\right)
$$




$$
\begin{aligned}
& -32 p_{1}^{3}\left(299 p_{2}^{3}+180 p_{3}^{2}-864 p_{2} p_{4}\right)+288 p_{1}^{4}\left(57 p_{2} p_{3}-28 p_{5}\right) \\
& -1536 p_{1}^{2}\left(17 p_{2}^{2} p_{3}-6 p_{3} p_{4}+6 p_{2} p_{5}\right) \\
& +512\left(9 p_{2}^{3} p_{3}-80 p_{3}^{3}+180 p_{2} p_{3} p_{4}-108 p_{2}^{2} p_{5}\right) \\
& +768 p_{1}\left(15 p_{2}^{4}-28 p_{2} p_{3}^{2}+21 p_{2}^{2} p_{4}-90 p 4^{2}+96 p_{3} p_{5}\right)
\end{aligned}
$$

suitably, we have

$$
\begin{aligned}
8847360 \Delta_{3}= & \left(768 p_{1}^{6} p_{3}-29 p_{1}^{9}\right)+\left(27648 p_{1}^{3} p_{2} p_{4}-5328 p_{4} p_{1}^{5}\right) \\
& +\left(-78 p_{1}^{7} p_{2}+16416 p_{1}^{4} p_{2} p_{3}\right)+1536 p_{1}^{2}\left(-17 p_{2}^{2} p_{3}+6 p_{3} p_{4}\right) \\
& +\left(73728 p_{1} p_{3} p_{5}-9216 p_{1}^{2} p_{2} p_{5}\right)+\left(92160 p_{2} p_{3} p_{4}-21504 p_{1} p_{2} p_{3}^{2}\right) \\
& +768 p_{1}\left(21 p_{2}^{2} p_{4}-90 p_{4}^{2}\right)+512\left(9 p_{2}^{3} p_{3}-108 p_{2}^{2} p_{5}\right) \\
& +\left(11520 p_{1} p_{2}^{4}-9568 p_{1}^{3} p_{2}^{3}\right) \\
& +\left(-1224 p_{1}^{5} p_{2}^{2}-5760 p_{3}^{2} p_{1}^{3}-8064 p_{1}^{4} p_{5}-40960 p_{3}^{3}\right)
\end{aligned}
$$

Using triangle inequality in (3.23) and applying Lemmas 1 and 4, we have

$$
\left|\Delta_{3}\right| \leq \frac{3720606449+33460224 \sqrt{717}+2942568 \sqrt{51737}}{5622886080} \approx 0.940065 .
$$

Now using the triangle inequality and substituting the bounds on initial coefficients $\left|a_{i}\right|(i=4,5,6,7)$ and $\left|\Delta_{j}\right|(j=1,2,3)$, we have

$$
\begin{aligned}
\left|H_{4,1}(f)\right| & \leq\left|a_{7}\right|\left|H_{3,1}(f)\right|+\left|a_{6}\right|\left|\Delta_{1}\right|+\left|a_{5}\right|\left|\Delta_{2}\right|+\left|a_{4}\right|\left|\Delta_{3}\right| \\
& \leq 1.93977 .
\end{aligned}
$$

Thus, we have the following:

Theorem 4. Let $f(z)=z+a_{2} z^{2}+a_{3} z^{3}+\cdots \in \mathcal{S}_{q}^{*}$. Then $\left|H_{4,1}(f)\right| \leq 1.93977$.

\section{ACKNOWLEDGEMENT}

The first author was supported by the Basic Science Research Program through the National Research Foundation of Korea (NRF) funded by the Ministry of Education, Science and Technology (No. 2019R1I1A3A01050861). The part of this work was completed during the second author's visit to the Department of Applied Mathematics, Pukyong National University (PKNU), Busan, South, Korea. The second author is thankful to PKNU for the facilities and hospitality provided during his stay in S. Korea. The second author also express his gratitude to the Principal of Ramanujan College for his encouragement, support and granting permission to visit PKNU (9-30 June 2019). 


\section{REFERENCES}

[1] R. M. Ali, "Coefficients of the inverse of strongly starlike functions," Bull. Malays. Math. Sci. Soc. (2), vol. 26, no. 1, pp. 63-71, 2003, in memory of Professor Dr. Mohamad Rashidi Bin Md. Razali.

[2] M. Arif, L. Rani, M. Raza, and P. Zaprawa, "Fourth Hankel determinant for the family of functions with bounded turning," Bull. Korean Math. Soc., vol. 55, no. 6, pp. 1703-1711, 2018, doi: 10.4134/BKMS.b170994. [Online]. Available: https://doi.org/10.4134/BKMS.b170994

[3] M. Çağlar, E. Deniz, and H. M. Srivastava, "Second Hankel determinant for certain subclasses of bi-univalent functions," Turkish J. Math., vol. 41, no. 3, pp. 694-706, 2017, doi: 10.3906/mat-1602-25. [Online]. Available: https://doi.org/10.3906/mat-1602-25

[4] U. Grenander and G. Szegö, Toeplitz forms and their applications, ser. California Monographs in Mathematical Sciences. University of California Press, Berkeley-Los Angeles, 1958.

[5] H. Güney, G. Murugusundaramoorthy, and H. M. Srivastava, "The second hankel determinant for a certain class of bi-close-to-convex functions," Results Math., vol. 14, no. 3, p. 13, 2019, doi: 10.1007/s00009-016-0829-y. [Online]. Available: https://doi.org/10.1007/s00009-016-0829-y

[6] W. K. Hayman, "On the second Hankel determinant of mean univalent functions," Proc. London Math. Soc. (3), vol. 18, pp. 77-94, 1968, doi: 10.1112/plms/s3-18.1.77. [Online]. Available: https://doi.org/10.1112/plms/s3-18.1.77

[7] A. Janteng, S. A. Halim, and M. Darus, "Hankel determinant for starlike and convex functions," Int. J. Math. Anal. (Ruse), vol. 1, no. 13-16, pp. 619-625, 2007.

[8] B. a. Kowalczyk, A. Lecko, and Y. J. Sim, "The sharp bound for the Hankel determinant of the third kind for convex functions," Bull. Aust. Math. Soc., vol. 97, no. 3, pp. 435-445, 2018, doi: 10.1017/S0004972717001125. [Online]. Available: https://doi.org/10.1017/S0004972717001125

[9] B. Kowalczyk, A. Lecko, M. Lecko, and Y. J. Sim, "The sharp bound of the third Hankel determinant for some classes of analytic functions," Bull. Korean Math. Soc., vol. 55, no. 6, pp. 1859-1868, 2018, doi: 10.4134/BKMS.b171122. [Online]. Available: https://doi.org/10.4134/BKMS.b171122

[10] V. Kumar, N. E. Cho, V. Ravichandran, and H. M. Srivastava, "Sharp coefficient bounds for starlike functions associated with the Bell numbers," Math. Slovaca, vol. 69, no. 5, pp. 1053-1064, 2019, doi: 10.1515/ms-2017-0289. [Online]. Available: https://doi.org/10.1515/ms-2017-0289

[11] O. S. Kwon, A. Lecko, and Y. J. Sim, "The bound of the Hankel determinant of the third kind for starlike functions," Bull. Malays. Math. Sci. Soc., vol. 42, no. 2, pp. 767-780, 2019, doi: 10.1007/s40840-018-0683-0. [Online]. Available: https://doi.org/10.1007/s40840-018-0683-0

[12] A. Lecko, Y. J. Sim, and B. Śmiarowska, "The sharp bound of the Hankel determinant of the third kind for starlike functions of order 1/2," Complex Anal. Oper. Theory, vol. 13, no. 5, pp. 2231-2238, 2019, doi: 10.1007/s11785-018-0819-0. [Online]. Available: https://doi.org/10.1007/s11785-018-0819-0

[13] S. K. Lee, V. Ravichandran, and S. Supramaniam, "Bounds for the second Hankel determinant of certain univalent functions," J. Inequal. Appl., vol. 2013, no. 281, p. 17, 2013, doi: 10.1186/1029242X-2013-281. [Online]. Available: https://doi.org/10.1186/1029-242X-2013-281

[14] C. R. Leverenz, "Hermitian forms in function theory," Trans. Amer. Math. Soc., vol. 286, no. 2, pp. 675-688, 1984, doi: 10.2307/1999815. [Online]. Available: https://doi.org/10.2307/1999815

[15] R. J. Libera and E. J. Zlotkiewicz, "Early coefficients of the inverse of a regular convex function," Proc. Amer. Math. Soc., vol. 85, no. 2, pp. 225-230, 1982, doi: 10.2307/2044286. [Online]. Available: https://doi.org/10.2307/2044286

[16] S. Mahmood, H. M. Srivastava, N. Khan, Q. Z. Ahmad, B. Khan, and I. Ali, "Upper bound of the third hankel determinant for a subclass of $q$-starlike functions," Symmetry, 
vol. 11(3), no. 337, pp. 1-13, 2019, doi: 10.1007/s00009-016-0829-y. [Online]. Available: https://doi.org/10.1007/s00009-016-0829-y

[17] M. Obradović and S. Ponnusamy, "Starlike and close-to-convex functions defined by differential inequalities," Appl. Math. Comput., vol. 215, no. 7, pp. 2642-2648, 2009, doi: 10.1016/j.amc.2009.09.004. [Online]. Available: https://doi.org/10.1016/j.amc.2009.09.004

[18] M. Obradović, S. Ponnusamy, and K.-J. Wirths, "Geometric studies on the class $\mathcal{U}(\lambda), " B u l l$. Malays. Math. Sci. Soc., vol. 39, no. 3, pp. 1259-1284, 2016, doi: 10.1007/s40840-015-0263-5. [Online]. Available: https://doi.org/10.1007/s40840-015-0263-5

[19] M. Obradović, S. Ponnusamy, and K.-J. Wirths, "Logarithmic coefficients and a coefficient conjecture for univalent functions," Monatsh. Math., vol. 185, no. 3, pp. 489-501, 2018, doi: 10.1007/s00605-017-1024-3. [Online]. Available: https://doi.org/10.1007/s00605-017-1024-3

[20] R. Ohno and T. Sugawa, "Coefficient estimates of analytic endomorphisms of the unit disk fixing a point with applications to concave functions," Kyoto J. Math., vol. 58, no. 2, pp. 227-241, 2018, doi: 10.1215/21562261-2017-0015. [Online]. Available: https://doi.org/10.1215/21562261-2017-0015

[21] C. Pommerenke, "On the coefficients and Hankel determinants of univalent functions," J. London Math. Soc., vol. 41, pp. 111-122, 1966, doi: 10.1112/jlms/s1-41.1.111. [Online]. Available: https://doi.org/10.1112/jlms/s1-41.1.111

[22] S. Ponnusamy and K.-J. Wirths, "Coefficient problems on the class $U(\lambda)$," Probl. Anal. Issues Anal., vol. 7(25), no. 1, pp. 87-103, 2018, doi: 10.15393/j3.art.2018.4730. [Online]. Available: https://doi.org/10.15393/j3.art.2018.4730

[23] R. K. Raina and J. Sokół, "On coefficient estimates for a certain class of starlike functions," Hacet. J. Math. Stat., vol. 44, no. 6, pp. 1427-1433, 2015.

[24] R. K. Raina and J. Sokół, "Some properties related to a certain class of starlike functions," $C . R$ Math. Acad. Sci. Paris, vol. 353, no. 11, pp. 973-978, 2015, doi: 10.1016/j.crma.2015.09.011. [Online]. Available: https://doi.org/10.1016/j.crma.2015.09.011

[25] V. Ravichandran and S. Verma, "Bound for the fifth coefficient of certain starlike functions," $C$. R. Math. Acad. Sci. Paris, vol. 353, no. 6, pp. 505-510, 2015, doi: 10.1016/j.crma.2015.03.003. [Online]. Available: https://doi.org/10.1016/j.crma.2015.03.003

[26] L. Shi, H. M. Srivastava, M. Arif, and S. Hussain, "An investigation of the third Hankel determinant problem for certain subfamilies of univalent functions involving the exponential function," Symmetry, vol. 11(5), no. 598, pp. 1-14, 2019, doi: 10.3390/sym11050598. [Online]. Available: https://doi.org/10.3390/sym11050598

[27] L. Shi, Q. Khan, G. Srivastava, J.-L. Liu, and M. Arif, "A study of multivalent q-starlike functions connected with circular domain," Mathematics, vol. 7(8), no. 670, pp. 1-12, 2019, doi: 10.3390/math7080670. [Online]. Available: http://dx.doi.org/10.3390/math7080670

[28] H. M. Srivastava, Q. Z. Ahmad, M. Darus, B. Khan, N. Khan, Z. N., and H. H. Shah, "Upper bound of the third hankel determinant for a subclass of close-to-convex functions associated with the lemniscate of bernoulli," Mathematics, vol. 7(9), no. 848, pp. 1-10, 2019, doi: 10.1007/s13398-019-00713-5. [Online]. Available: https://doi.org/10.1007/s13398-019-00713-5

[29] H. M. Srivastava, Q. Z. Ahmad, N. Khan, N. Khan, and B. Khan, "Hankel and toeplitz determinants for a subclass of $q$-starlike functions associated with a general conic domain," Mathematics, vol. 7(2), no. 181, pp. 1-15, 2019, doi: 10.1007/s13398-019-00713-5. [Online]. Available: https://doi.org/10.1007/s13398-019-00713-5

[30] H. M. Srivastava, c. Altinkaya, and S. Yalçin, "Hankel determinant for a subclass of bi-univalent functions defined by using a symmetric q-derivative operator," Filomat, vol. 32, no. 2, pp. 503516, 2018, doi: 10.2298/fil1802503s. [Online]. Available: https://doi.org/10.2298/fil1802503s

[31] H. M. Srivastava, N. Raza, E. S. A. AbuJarad, G. Srivastava, and M. H. AbuJarad, "Fekete-Szegö inequality for classes of (p, q)-Starlike and (p, q)-convex functions," Rev. R. Acad. 
Cienc. Exactas Fís. Nat. Ser. A Mat. RACSAM, vol. 113, no. 4, pp. 3563-3584, 2019, doi: 10.1007/s13398-019-00713-5. [Online]. Available: https://doi.org/10.1007/s13398-019-00713-5

[32] A. Vasudevarao and H. Yanagihara, "On the growth of analytic functions in the class $\mathcal{U}(\lambda)$, Comput. Methods Funct. Theory, vol. 13, no. 4, pp. 613-634, 2013, doi: 10.1007/s40315-013-0045-8. [Online]. Available: https://doi.org/10.1007/s40315-013-0045-8

[33] P. Zaprawa, "Third Hankel determinants for subclasses of univalent functions," Mediterr. J. Math., vol. 14, no. 1, p. 10, 2017, doi: 10.1007/s00009-016-0829-y. [Online]. Available: https://doi.org/10.1007/s00009-016-0829-y

\section{Authors' addresses}

\section{Nak Eun Cho}

Department of Applied Mathematics, Pukyong National University, Busan 48513, Republic of Korea

E-mail address: necho@pknu.ac.kr

\section{Virendra Kumar}

Department of Mathematics, Ramanujan College, University of Delhi, Kalkaji, New Delhi 110019, India

E-mail address: vktmaths@yahoo. in 